\title{
A Design of Digital Handheld Terminal in Wireless Data Acquisition System
}

\author{
Xingguo SUN \\ Key Lab of Intelligent Computing and Signal Processing of \\ Ministry of Education, Anhui University \\ Hefei, China \\ Shouxian WEN \\ Key Lab of Intelligent Computing and Signal Processing of \\ Ministry of Education, Anhui University \\ Hefei, China
}

\begin{abstract}
In order to make data collection more convenient, quick and accurate in working site in the existing wireless data acquisition system, one kind of multi-function handheld terminal is designed which combines the wireless and wired data acquisition. The paper adopts Atmega128 of ATMEL as microcontroller, nRF905 of Nordic as data transmission module. It designs power management, liquid-crystal display and data storage circuits. The results of practical test show that the handheld terminal can be applied to all kinds of wireless data acquisition networks, and it has several advantages like easy to carry, large data storage capacity, rich function and low cost.
\end{abstract}

Keywords-DS18B20 ; NRF905 ; Handheld terminal ; Data acquisition system

\section{INTRODUCTION}

With the popularization of the large amount, cheap and highly integrated wireless module and the rapid development of wireless communication technology, the wireless data acquisition system has been widely used in industry, agriculture, medical and other fields. The existing wireless data acquisition system is composed of a convergent node, basic nodes and the server. The convergent node exchanges information with basic nodes by the radio frequency (RF) module or Zigbee module[1-4].Basic nodes collect information by the digital sensors and send it to the converge node in the form of packet. The data which is gathered by the converge node can be sent to the local server[5] or be transmitted to the remote server by GPRS [6]or GSM[7]. This way need to pay for the expensive network cost, and can not collect data conveniently, quickly and accurately[2].

Based on the analysis above, this paper proposes a design scheme of handheld terminal. nRF905 is chosen as communication module which can realize remote transmission. We design the routing protocol which realizes automatic searching and manual setting. It makes the handheld terminal collect data information reliably in any effective area of the wireless network coverage and then display the data on LCD. Handheld terminal has the USB interface circuit which can be directly connected to the computer and allow computer to

\author{
Lei XU \\ Key Lab of Intelligent Computing and Signal Processing of \\ Ministry of Education, Anhui University \\ Hefei, China \\ Xiaohui LI* \\ Key Lab of Intelligent Computing and Signal Processing of \\ Ministry of Education, Anhui University \\ Hefei, China
}

receive or send commands and data. In addition, it also supports the wired data acquisition, data printing.

\section{APPLICATION SCENARIO}

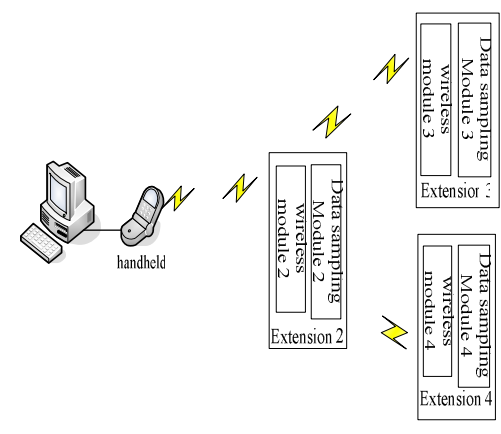

Figure 1. Network Structure

Figure 1 illustrates the network structure of application scenario. The wireless data acquisition system can be divided into three parts: extension, handheld terminal and the server.

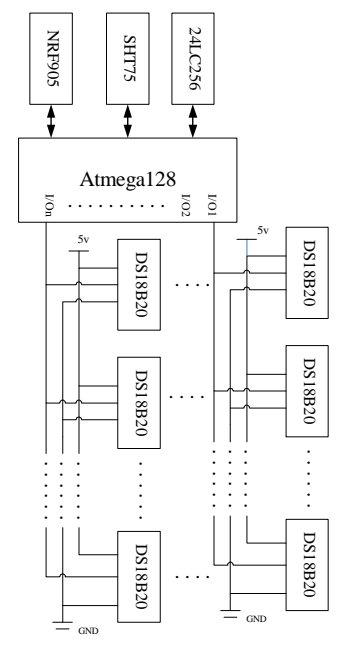

Figure 2. Extension Structure

*Corresponding author.

Email address: xhli@ahu.edu.cn (Xiaohui LI). 
Extension structure is shown in Figure 2. According to the received instructions, the extension samples the data of temperature and humidity and then transmits to the handheld. We use DS18B20 and SHT75 as temperature and humidity sensors respectively. The circuit of temperature data sampling adopts 1-Wire single-bus mode, so multiple temperature sensors can be mounted on each I/O port. In order to identify the physical location of DS18B20 and to facilitate the server data management, the corresponding layer information is written into the DS18B20 alarm trigger register[8]. When it's power on, the extension reads DS18B20 ROM serial number and the layer information in alarm trigger register and then store ROM serial number in 24LC256 according to the different I/O ports and layer information. The physical location of DS18B20 can be determined according to the ROM serial number's store address to realize the effective data management.

Handheld terminal works as a convergent node in the wireless network .It collects the data of extension through the nRF905 and transmits it to the computer through the USB interface. In the working site, handheld terminal can search extension through the routing discovery menu and establish a routing table. According to the actual situation, we input an extension's address. The handheld terminal will set up a path according to the routing table information to realize communication with the extension. The data will be displayed on the LCD after processed. Handheld terminal has data storage, data printing and cable numbering functions. Cable numbering can facilitate system maintenance. When an $\mathrm{I} / \mathrm{O}$ port of a certain extension appears bad points, these points will be identified through measuring temperature. Handheld terminal ignores these bad points and rewrites layer information of other sensors through cable numbering function.

In addition, handheld terminal can collect temperature and humidity data using wired way through link it to cable together directly in the following two cases. One case is that some small temperature and humidity acquisition systems will not use wireless extensions in order to reduce the cost; the other case is that the geographical position is too scattered to suitable for networking.

The server transmits specified instructions to handheld terminal through the USB interface. The handheld terminal sends these instructions to the corresponding extension node through the wireless network. With this, the data can be gathered and passed back to the server. The server completes data analysis and management.

\section{DESIGN OF HARDWARE CIRCUIT}

Hardware of handheld terminal consists of several parts, which is shown in Fig. 3.

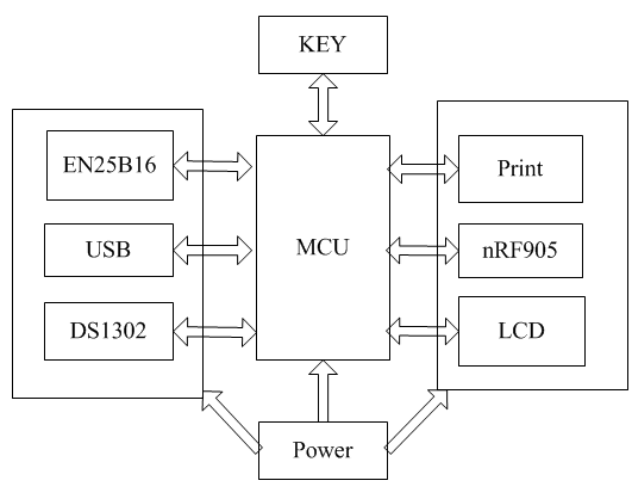

Figure 3. Hardware Structure

\section{A. Control Module}

This paper chooses a low power consumption, high performance microprocessor Atmega128 as main control chip, which makes the handheld terminal processing faster and working longer. This module is a core control unit of handheld terminal. It obtains instructions from the keyboard and coordinates the whole system.

\section{B. Wireless Module}

The wireless transceiver module adopts radio transceiver single-chip nRF905 which works in the $433 / 868 / 915 \mathrm{MHz}$ of ISM (Industrial Scientific Medical). The ShockBurst TM feature automatically handles preamble and the CRC (Cyclical Redundancy Check)[9]. In addition, the nRF905 has stand by and power down modes which can save more energy. The Atmega128 connects with nRF905 through SPI. When transmitting the data, the Atmega128 just sends the configuration register information, the receiver address and the transmitted data to nRF905, then nRF905 packages and sends all data (plus preamble and the CRC). When receiving the data, nRF905 detects the carrier and matches the address automatically. The data is received correctly and the preamble, address and CRC are removed, and then the data is transmitted to Atmega128 through SPI.

\section{Power Module}

Lithium Battery is for power supply of the handheld. The charge management chip is CN3052. In the processing of charging, the system automatically switches to an external power to ensure that the system can still work. Once the charge cycle has terminated, the charging indicator light on the handheld will turn off. Due to the working voltage of printer module is $5 \mathrm{~V}$, while the other modules operating voltage is 3.3V. The proposed scheme chooses ME6211A33 as 3.3V voltage regulator and LTC1700 as 5V high-current output voltage regulator.

\section{Function Module}

The handheld terminal uses CP2102 to achieve a highspeed interface to realize communication between the computer and the single-chip Atmega128. We use a $128 \times 128$ lattice LCD screen as display module and use $2 \times 2$ matrix 
keyboard to realize man-machine information exchange. EN25B16 is a 2M bytes external storage flash and the data can be stored in it. The DS1302 will support time information. In addition, a printer module RD-OEM57V1 is embedded in the handheld terminal which supports data printing.

\section{SOFTWARE DESIGN}

The embedded software of handheld terminal is programmed by C Language with the development of IAR Embedded Workbench, and adopts module program structure design. The main tasks of software include data processing, ask sequencing and job scheduling. When the program stars, handheld will initialize the system, including LCD, nRF905, the detecting of battery energy and the corresponding registers, then enter into the initial interface. User can select menu to complete the corresponding function by keyboard. Work flow diagram of handheld is shown in Figure. 4

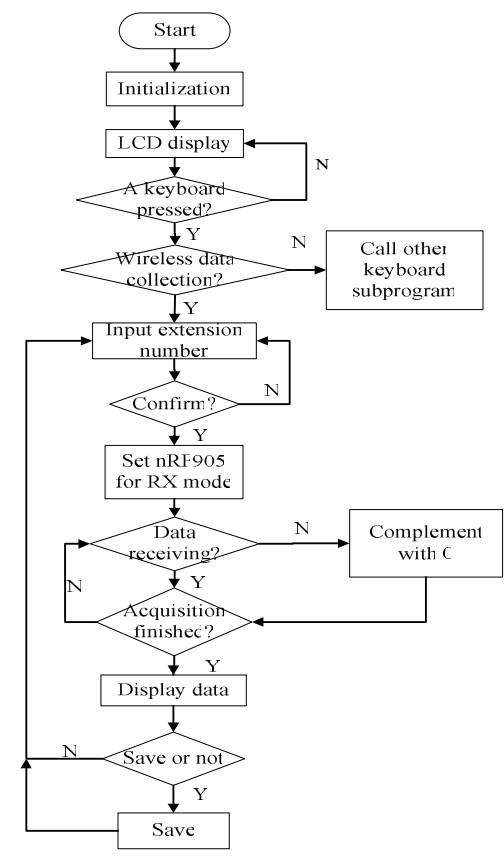

Figure 4. The flow chart of the software

The communication protocol of the system bases on the master-slave structure. The extension can not initiatively launch communication, and it only has a passive response ability. In the system, each extension has two addresses: one is physical address; the other is network address. The physical address of all the extensions is $0 x F F$, but the network address is unique. When the extension receives instruction, it will determine whether the address correctly or not. Only the address is correct, the extension will execute instructions and return the data. Data frames format of nRF905 is as following table I .

TABLE I. DATA FRAME FoRMAT

\begin{tabular}{|c|c|c|c|c|}
\hline Type & Route & Route_Depth & Route_Direction & Data \\
\hline
\end{tabular}

Type code is 1 byte, it is used to distinguish the different operations. Route is 5 bytes. It represents routing information which is obtained from the routing table. The first byte is the source address, the next byte is the destination address, the last 3 bytes are the relay address; the Route_Depth and Route_Direction are all 1 byte which are used to point to the next address. Data represents the transmitted data. If the data is less than 10 bytes, the remaining bits are complemented with 0 .

When the handheld failed to send message for 10 consecutive times, we can manually change the routing or alternatively enter the routing discovery phase. The wireless network uses dynamic routing mechanism[10]. Each frame of data contains the routing information from the source address to the destination address. The extension need not do any routing operations. It only transmits the data to the next extension, according to the routing information in the packet.

During the routing discovery, the handheld terminal broadcasts RREQ packet to all extensions. If the received RREQ extension address is the destination address of routing requesting, the extension will return RREP packet to the handheld terminal, or it will forward RREQ packet. The RREP packet carries the routing information from the source address to the destination address. The flow diagram of route is shown in Figure 5.

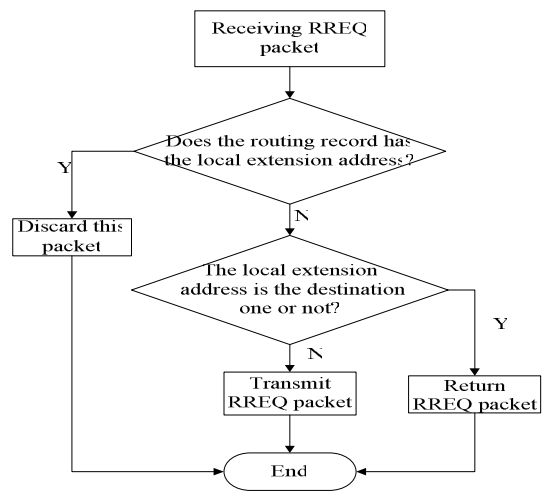

Figure 5. The flow diagram of route

\section{THE EXPERIMENTAL RESULTS}

Handheld terminal collects temperature and humidity information of sensors which are mounted on NO.3 extension through NO.2 relay extension. NO.3 extension hangs two cables. There are six DS18B20 on each cable. The data results are shown in Figure 6.
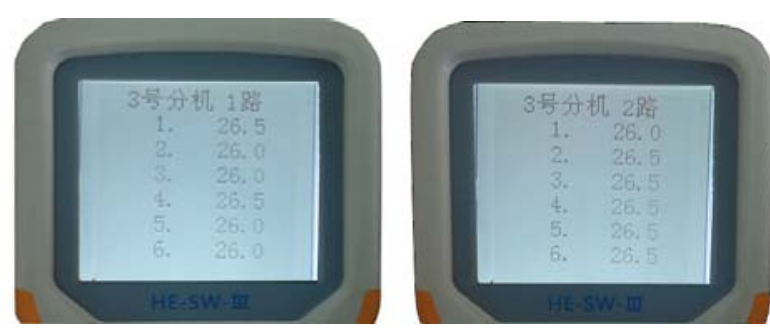

Figure 6. Experimental results 


\section{CONCLUSION}

This handheld terminal combines the wireless and wired data acquisition. It can detect data of working site by wireless way and collect temperature and humidity information by linking to cable directly. The handheld terminals can still work normally in the environment where working site has various forms or extension structure is complicate. In addition, handheld terminal can also connect with computer and allow PC to realize real-time detection, data backup, data sharing and so on.

\section{ACKNOWLEDGEMENT}

This project is supported by the National Natural Science Foundation of China (No. 60972040), the Anhui Provincial Natural Science Foundation (No. 11040606Q06), the Provincial Project of Natural Science Research for Colleges and Universities of Anhui Province of China (No. KJ2012A003) and the 211 Project of Anhui University.

\section{REFERENCE}

[1] Chang Chun-bo. Design and Realization of the Wireless Grain Information Monitoring Syestem of Low Power Consumption[D]. Taiyuan: Taiyuan University of Technology, 2007
[2] Wang Quan, Chen Jia-lin, Xie Ying, Dai Jian-bo, Liu Chao. "Design and Implementation of Industrial Field ZigBee handheld Controller", Journal of Microcomputer Information, Vol.25,No.5-2,2009

[3] Wang Quan,Wang Jing-chuan,Wei Min,Chen Jia-lin. "Design and Implementation of Industrial Wireless Handheld Operator”,Journal of Industrial Control Computer, Vol.22,No.6,2009

[4] Zheng Li-hua,Ling Qing-nian,Li Lu-wei. "The Design of Handset Based on Industrial Wireless Network”,Journal of Electrical Measurement \& Instrumentation,Vol.47,NO.536A, 2010

[5] Jiang Xiao, Bei Jiang, Kan Jiang-ming, "Design of Wirelss Temperature and Humidity Monitoring System of the Intelligent Greenhouse,”ICCET. Beijing, pp. 59-63 V3.April 2010

[6] Rui Zhao, Kaixue Yao, Meng Wei. "The Research and Design of Engine Room Temperature and Humidity Remote Monitoring System Based on GPRS,”IHMSC.Guiyang.pp.219-222. August 2011

[7] Jifeng Ding, Jiyin Zhao, Biao Ma. "Remote monitoring system of temperature and humidity based on GSM,"CISP.Dalian, pp.1-4. October 2009

[8] Xu lei, Zhang Hong-wei, Li Xiao-hui and Wu Xian-liang. “A Design of Wireless Temperature and Humidity Monitoring System,”ICCT. Hefei, pp. 13-16. November 2010

[9] Yingli Zhu, Wanghui Zeng and Lingqing Xie. "Design of Monitoring System for Coal Mine Safty Based on MSP430 and nRF905,’ISIE. Nanchang, pp. 98-101.October 2011

[10] Dou Niu, Yan Zhang, Yanjuan Zhao, Mei Yang, "Research on Routing Protocols in Ad Hoc Networks,” WNIS. Jilin, pp.27-30. October 2009 